\title{
Review Article \\ ctDNA Detection in Microfluidic Platform: A Promising Biomarker for Personalized Cancer Chemotherapy
}

\author{
Samla Gauri $(D)$ and Mohd R. Ahmad $(D)$ \\ Division of Control and Mechatronics Engineering, School of Electrical Engineering, Faculty of Engineering, \\ Universiti Teknologi Malaysia, 81310 Skudai, Johor, Malaysia \\ Correspondence should be addressed to Mohd R. Ahmad; mdridzuan@utm.my
}

Received 27 August 2019; Accepted 10 December 2019; Published 8 April 2020

Academic Editor: Luca De Stefano

Copyright (c) 2020 Samla Gauri and Mohd R. Ahmad. This is an open access article distributed under the Creative Commons Attribution License, which permits unrestricted use, distribution, and reproduction in any medium, provided the original work is properly cited.

\begin{abstract}
Early detection and characterization of circulating tumor DNA (ctDNA) can reveal mint of comprehensive biological insights from indicating the presence of tumor, identifying mutational changes of malignant cells, and allowing precision or targeted therapy together with monitoring disease progression, treatment resistance, and relapse of the disease. Apart from these, one of the greatest axiomatic implications of ctDNA detection is that it provides a new shed of light as noninvasive liquid biopsy as a replaceable procedure of surgical tumor biopsy. Despite the tremendous potential of ctDNA in cancer research, there remains a paucity of quantitative study on ctDNA detection and analysis. The majority of previously published microfluidic-based studies have focused on circulating tumor cell (CTC) detection and have failed to address the potential of ctDNA. The studies on microfluidic ctDNA detection are not consistent might be due to the complexity of ctDNA isolation as they present in low concentration in blood plasma. Researchers need to leverage the ability of microfluidic system for ctDNA analysis so that the significant enigma about cancer can be resolved effectively. This study, therefore, highlights the importance of ctDNA as cancer biomarker for liquid biopsy and provides an overview of the current laboratory as well as microfluidic techniques for ctDNA detection. This paper also attempts to show the emergence of new strands of microfluidic ctDNA detection and analysis for personalized cancer chemotherapy.
\end{abstract}

\section{Introduction}

A biomarker is a biomolecule found in body fluids or tissues that describes a normal or abnormal biological state of condition or disease. The World Health Organization [1] defined biomarker as biological substance, structure, or process that can be measured in the body for disease prediction [2]. Generally, the potential biomolecules as biomarkers are proteins (enzyme or receptor), peptides, nuclei acids (DNA, RNA), antibodies, and alterations such as gene expressions, proteomic signatures, metabolomic signatures, and cell mutations [3]. In cancer research, cancer biomarkers are biomolecules that secreted by tumor and responding specifically due to the presence of tumor. Recently, the most studied and effectively detectable cancer biomarkers are circulating tumor cells (CTCs) and circulating tumor DNAs (ctDNA). These biomarkers can be identified in the circulation of whole blood, serum, and plasma or in other body fluid such as urine and saliva [3]. Apart from predicting disease as a prognostic biomarker, it also measures the risk of cancer progression, response towards treatments, and pharmacogenomic response (response of drug by cells). This term is also known as a diagnostic biomarker used in personalized medicine where precise treatment or therapy can be provided without adverse reaction $[2,4]$.

Circulating tumor cells (CTCs) are cells that originate and disgorge from primary tumor, recurrences, or metastases that are circulating freely in the bloodstream [5]. They have specific genetic characteristic of the primary tumor and metastasize to various part of the organ even in primary stage [6]. Based on several reviews, CTCs can be identified at the early stage of tumor growth and this can be therapeutic biomarker for treatment evaluation as well as detecting metastatic relapse of a particular disease or tumor $[7,8]$. CTCs may 

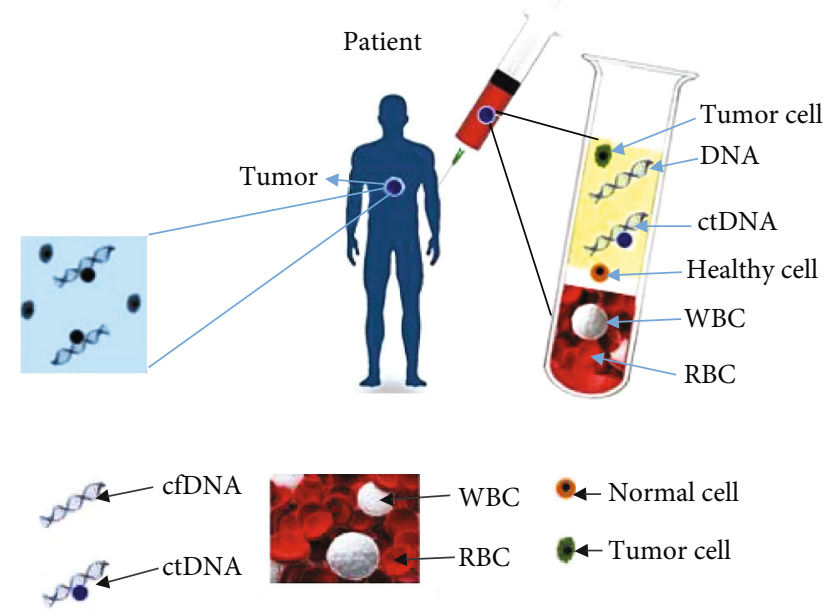

FIgURE 1: Components in blood sample of a cancer patient after the centrifugation process. White blood cells (WBC) and red blood cells (RBC) can be found in red blood, while normal cells, tumor cells, cfDNA, and ctDNA can be found in plasma.

release from various tumor of various part of the organ which varies in nature. Thus, CTCs can display heterogeneous cells that exuviate from primary tumor which an additional analysis is needed to study the characteristic and location of the primary tumor. Recently, CTCs isolated based on promising noninvasive liquid biopsy technique and have been validated as potential biomarkers in diversity of cancers including lung, colorectal, breast, and prostate [9]. However, the concentration of CTCs in the bloodstream is very low as such 1 in $10^{9}$ of blood cells and only a particular number of CTCs able to generate metastases. Accordingly, there is a need for further analysis to characterize metastatic and nonmetastatic CTCs for appropriate precise therapy $[5,10]$.

Circulating cell-free DNA (cfDNA) is degraded small DNA fragments found circulating freely in the bloodstream (plasma and serum) and other body fluids. It is mostly double-stranded extracellular molecules of DNA with smaller fragments around $70 \mathrm{bp}$ to $200 \mathrm{bp}$ which do not necessarily originate from the tumor $[11,12]$. Based on earlier studies, researchers found that the presence of cfDNA is significantly higher in cancer patients compared to healthy patients. The elevated number of cfDNA contains a diluted number of tumor-specific mutation which also known as circulating tumor DNA (ctDNA) [13]. ctDNA is a biomarker released from cancerous cells and tumors as they grow and replaced by new ones. The number of ctDNA difference depends on tumor type, location, and tumor cascade. The overall picture of CTCs, cfDNA, and ctDNA is shown in Figure 1.

Analysis of ctDNA provides revelation about heterogenous information for detecting tumor by liquid biopsy, providing more effective precision therapy and monitoring disease condition including nonhematological as well as progression [14]. Cancer can be categorized as an incommensurable disease where the molecular mechanism of tumor eventually responses over tumor-stage progression, treatment, or immune system changes during therapy. Almost all chemotherapies have the resistance criteria and lead to tumor clonal evolution for the duration of disease progres-

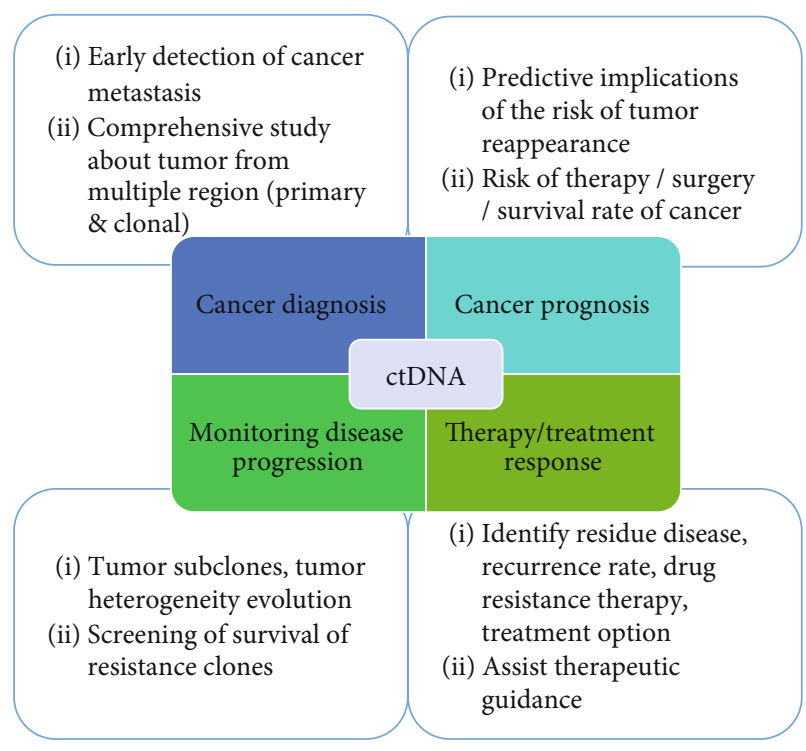

FIgURe 2: The potential clinical benefits of ctDNA detection as a promising biomarker for cancer treatment and management.

sion [15]. As ctDNA is released from multiple tumor regions including primary and clonal, the analysis provides voluminous information regarding tumor heterogeneity and clonal evolution. A great deal of previous research has recognized the feasibility of ctDNA in detection of tumor residual, survival of resistant clones, and drug resistance over treatment and predicts emerging subclones before recurrence of tumor [16-18]. Moreover, early detection of metastasis throughout tumor progression is possible by ctDNA. ctDNA alteration and the escalating number of ctDNA in early-stage also predict the probability of disease recurrence [19-21]. Some cross-sectional studies about ctDNA predicted the patient prognosis when treated with surgery, chemotherapy, or radiotherapy and risk of tumor reappearance [22, 23]. The study furthermore can be used to monitor treatment response rate for better cure management and therapeutic guidance [24, 25]. It has conclusively been shown that ctDNA detection is comparative to cancer stage. The detection rate is $100 \%$ for II-IV compared to stage I which is $50 \%$ for non-small-cell lung cancer (NSCLC) $[18,26]$. The inclusive potential clinical benefits of ctDNA detection are described in Figure 2.

Data from several sources have stated that it is easier to separate ctDNA than CTCs as ctDNA is more stable and higher in proportion in the bloodstream with high sensitivity. Only few CTCs are detectable in a single blood draw of $7.5 \mathrm{ml}$, whereas cfDNA including ctDNA is also released by apoptotic cells and tumor cells [21,27]. Their concentration is directly proportional to the tumor cascade [28]. In spite of that, further isolation procedure of methylation analysis is needed to well define tumor-specific cfDNA or particularly ctDNA. Methylation analysis is DNA chromosomal pattern study by the methyl group. The patterns of genome changes in methylated DNA show the exact tumor-specific DNA particularly ctDNA or biomarker identification $[29,30]$. The identification of suitable cancer biomarker hinges the pathway towards tangible personalized medicine. The escalating personalized medicine in revolutionizing healthcare enables 
to deliver the right treatment for the right person at the right time for disease diagnosis screening, monitoring tumor progression, and drug resistance reactions [31]. Analysis of ctDNA as cancer biomarker is amenable for noninvasive liquid biopsy which gives quick access to tumor sample rather than conventional tissue biopsy which needed surgical examination of tumor tissue. In spite of the fact that ctDNA highlighted many flourishing possibilities as well-validated prognostic and diagnostic biomarker in cancer research, yet the emergence of a high-sensitive and selective point-ofcare device to leverage the potential of ctDNA is still needed. Microfluidic platform as a healthcare device plays an essential role in addressing the needs for modern molecular technology in manipulating various analyses for DNA, proteins, and cells simultaneously or consecutively depending on requirements. Hence, this review summarizes the current laboratory liquid biopsy techniques, developed microfluidic liquid biopsy devices, and future trends for rapid detection and characterization of ctDNA in cancer patients.

\section{Methods of ctDNA Analysis}

Identification and analysis of ctDNA typically have been carried out in multiple laboratories which required large equipment and professional skills to handle the sample and it is considered time-consuming procedures. Consequently, ctDNA rapid detection and analysis was developed from the laboratory (macroscale analysis using a large volume of sample) to microfluidic techniques (microscale analysis using a small volume of sample). The development of this analysis is described in the following part.

\section{Laboratory Liquid Biopsy Techniques}

A biopsy is an investigation of cell or tissue taken surgically from tumor to study and confirm the presence, cause, and stage of a tumor or disease. Conventional tissue biopsy is clinically not worth considering for some diseases screening mainly for lung and brain cancers. Surgically removing part of tissue may cause of nerve injury and bleeding or disease spreading. Moreover, surgery during chemotherapy where the genome of the tumor cell is highly unstable for continuous monitoring of medication and tumor progress is unbearable [32]. In contrast, liquid biopsy where analysis is based on fluid sample such as blood offers noninvasive continuous heterogeneous and comprehensive disease observation technique. Diversity of required information about the type, stage of the tumor, and chemotherapy progress from personalized medicine can be accessible from time to time through detection of ctDNA by this liquid biopsy [33]. The required procedures for tissue biopsy and liquid biopsy are described as a comparison in Figures 3 and 4.

Current laboratory technique and analysis of ctDNA can be divided into four categories. They are sequencing, polymerase chain reaction (PCR) including Scorpion Amplification Refractory Mutation System (ARMS), beads, emulsions, amplification and magnetic analysis (BEAMing), and next-generation sequencing (NGS) $[32,34,35]$. PCR, realtime quantitative PCR (qPCR), digital PCR (dPCR), or

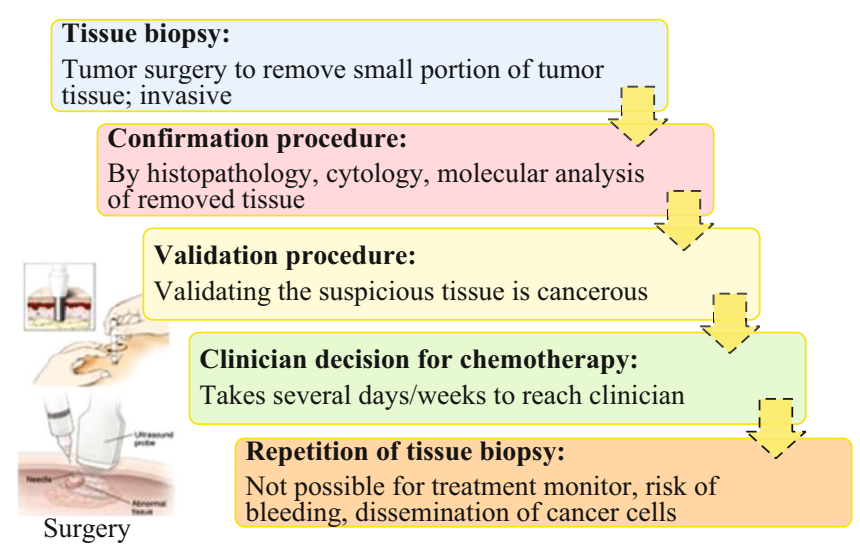

FIGURE 3: The overall procedures of tissue biopsy only provide a static tumoral snapshot of a specific time point and do not reflect dynamic changes that occur during cancer treatment.

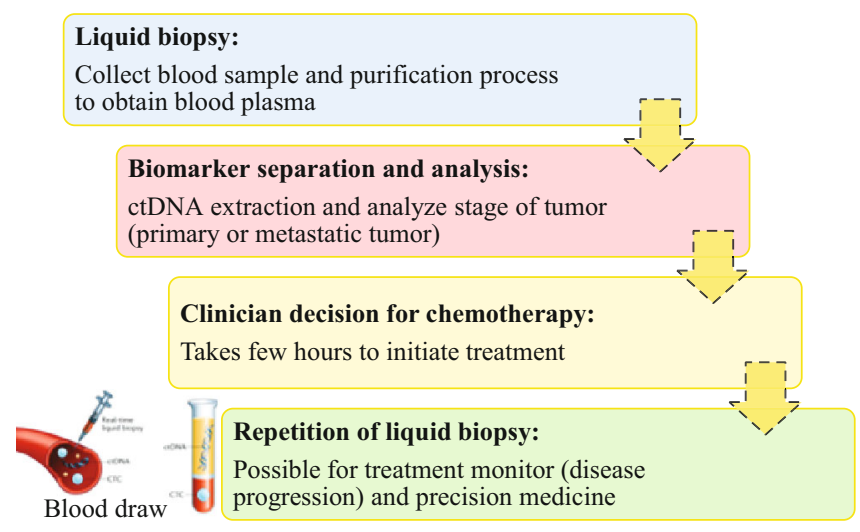

FIgURE 4: The overall process of liquid biopsy as noninvasive process that is possible to assess the dynamic tumoral changes by ctDNA analysis over the course of therapy and possibilities for early therapy before the tumor spreading.

improved version of droplet digital PCR (ddPCR) is modified from a conventional method to study genetic alteration, amplify, and detect rare known mutant DNA molecules as well as known allele-specific mutation [36]. PCR and qPCR have been the most widely used methods for quantification of nucleic acid. Nevertheless, they were considered as insensitive to detect low concentration of the mutant allele and the amplification initiates when only the required amount of targeted allele is present in the sample. Thus, researchers developed Scorpion ARMS integrated with real-time qPCR to detect low concentrated mutant allele $[37,38]$. Then, ddPCR was introduced where it amplified targeted DNA templates only from the whole sample and the presence of analytes sequence was shown through the positive signal indicator. This method was considered as high insensitive by reducing false-negative rates which are typically observed by qPCR-based techniques [39]. Similarly, ddPCR and BEAMing are also based on emulsion and amplification where isolation of required template into thousands of tiny droplets of the reaction chamber for amplification [39]. BEAMing with the same principle as ddPCR binds DNA to magnetic microbeads before emulsion into droplets. The 
method is based on sorting beads that contain cell mutation by using flow cytometry [40]. After the amplification process, magnetic microbeads are eluted out for purification of PCR product and mutation can be detected [41]. Although the detection capacity for various stages of cancer and sensitivity is quite high for Scorpion ARMS, ddPCR, and BEAMing, but these methods are only applicable for known mutations only and procedures are complex for clinical setting [42]. Numerous studies have presented the detection of ctDNA in cancer patient, but due to practical limitation in clinical application, sequencing and NGS methods were developed. These methods allowed ctDNA detection by parallel sequencing of hundreds of millions of DNA fragments from a single sample. The parallel sequencing is also used for simultaneous detection of varieties of potential mutations. NGS analysis also can be used to assist abundance of clinical decisionmaking from biomarker detection, analysis for diagnosis, prognosis, and monitoring of cancer treatments [43-46].

In an analytical study based on the mentioned current laboratory techniques, the percentage of detectable ctDNA in tumors varies according to stages as $49 \%$ to $78 \%$ of sensitivity in localized tumors while $86 \%$ to $100 \%$ of sensitivity in metastatic tumors [47]. Ishii and his group [48] detected drug resistance mechanism in epidermal growth factor receptor (EGFR) tyrosine kinase inhibitor (TKI) treatment for non-small-cell lung cancer (NSCLC). The analysis with $81.8 \%$ sensitivity plus $85.7 \%$ specificity was based on collected plasma (ctDNA) and tumor sample and conducted using droplet digital PCR (ddPCR). EGRF mutant in nonsmall-cell lung cancer (NSCLC) with analysis of $81 \%$ sensitivity and $85 \%$ specificity was detected by Gray et al. [24]. Another few studies presented ctDNA detection and confirmed the presence of colorectal cancer [49] and ctDAN of breast cancer [50]. Ashida et al. [51] successfully detected rare mutant DNA molecules and specified allele by PCR amplification. van Ginkel and his colleagues [52] identified ctDNA and specified rare mutational marker by ddPCR and BEAMing using a blood sample. Sequencing either whole genome sequencing (WGS) or whole exome sequencing (WES) is a comprehensive study of the tumor genome for rare mutation identification, therefore, assisting in clinical treatments $[35,53]$. Although WGS is used to identify malignant mutation, the changes in the primary tumor due to given therapy affect the subsequent analysis of the tumor genome. Similarly, the high-yield WES is used to detect rare disease and tumor cell mutation but is only applicable at later stage of tumor detection. The next-generation sequencing presented numerous advantages from reduced sequencing time, cost, and detail analysis of genome by simultaneous sequencing of millions of DNA molecules. The first modified method of sequencing is known as tagged-amplicon deep sequencing (TAm-Seq) which was used to identify the presence of metastatic mutations in patients with advanced ovarian cancer. Then, the capture-based deep sequencing method was introduced by Newman et al. [54] and developed together with Bratman et al. [26] to identify ctDNA in multiple mutations with comprehensive diagnostic information, used to monitor residual disease and detected single nucleotide variants. Then, this method was further improvised by
Newman and his group in [55] to digital error suppressionenhanced deep sequencing mainly to increase the sensitivity and specificity of ctDNA detection. Volik et al. [13] demonstrated Safe-Sequencing System (Safe-SeqS), based on amplicon approach for effective detection of rare variants and able to identify ctDNA in many tumor types by reducing the sequencing error rate. However, this required a sophisticated data analysis system with patient-specific optimization of amplicon for alteration such as somatic copy number and fragment rearrangement detection. In a follow-up study, WGS was used to analyse ctDNA in patients with metastatic breast cancer and treatment options were studied too. By the same token, WES was used for ctDNA detection in patients with advanced breast, ovarian, and lung cancer [56]. The summary of the laboratory method for ctDNA detection is shown in Table 1. Although these methods are sensitive in ctDNA detection and monitor disease progression, but they remain expensive and time-intensive and require sophisticated data analysis systems. Thus, clinical microfluidic device has shed a new light for rapid, cost-effective, and most importantly with simple workflow where this technique could be repeated anytime anywhere for molecular analysis as shown in Figure 5.

\section{Microfluidic Liquid Biopsy Techniques}

The past decade has seen the rapid development of microfluidic system for ranges of healthcare considerations. A single microfluidic chip is able to perform series of analysis or experiments conducted in an entire lab which requires several days to reveal the output. As the required sample and reagent volume is small, the microfluidic platform might be applicable for analysis of a single cell. Mostly, microfluidic device can be created as a portable, transportable, pocketsized tool and also diminutive bench top instrument. It would be a great supporting device to deliver the right care at the right time. However, there are some considerations in the development of the microfluidic devices for medical essential. The poor quality of analysis and result interpretation will directly lead to an inaccurate treatment option thus may affect patient's health. Although the raw specimen input to the device does not necessitate the centrifugation process, yet the quality of specimen and analytical technique play an imperative role to produce high-yield result.

There is a relatively small body of academic literature concerned with ctDNA detection via microfluidic. This is essentially due to insensitive detection of low concentrated ctDNA in the background of nonmutated DNA from normal cells. Major advances in microfluidic have revealed the emergence of application in molecular and biomedical for rapid analysis of clinical usage. Size and properties of ctDNA are one of the ideas to initiate the development of microfluidic ctDNA detection. Accordingly, electrokinetic trapping on microchannel was introduced which relies on the accumulation of charged ions by applied electric field forming the depletion of targeted analytes [57]. In a follow-up study, size-based micropillar structure on microchannel, microcolumn-packed separation, and microchannel with the electrophoretic system $[58,59]$ were developed for cfDNA extraction to achieve high yield 
TABLE 1: Laboratory methods for ctDNA detection to identify rare mutational marker and the presence of tumor.

\begin{tabular}{|c|c|c|c|}
\hline Method & Description & Limitations & References \\
\hline Whole genome sequencing & Deep sequencing of the genome & $\begin{array}{c}\text { Expensive, limited to SCNA } \\
\text { detection with low insensitivity }\end{array}$ & {$[53]$} \\
\hline Whole exome sequencing & Deep sequencing of the exome & Expensive and low insensitivity & [53] \\
\hline Next-generation sequencing & $\begin{array}{l}\text { Capture-based deep sequencing for detecting } \\
95 \% \text { of tumor in NSCLC. Detection specificity } \\
\text { of } 96 \% \text { for mutant allele fraction in } 100 \% \text { of } \\
\text { stages II-IV patients and } 50 \% \text { of stage I patients }\end{array}$ & $\begin{array}{l}\text { Less comprehensive than whole } \\
\text { genome/exome sequencing }\end{array}$ & {$[26,54]$} \\
\hline \multirow{5}{*}{ Digital or droplet digital PRC } & $\begin{array}{l}\text { Detect EGRF mutant in NSCLC with analysis } \\
\text { of } 81 \% \text { sensitivity and } 85 \% \text { specificity }\end{array}$ & \multirow{5}{*}{$\begin{array}{l}\text { Detection limit to a small } \\
\text { number of genomic positions } \\
\text { in the sample }\end{array}$} & {$[24]$} \\
\hline & $\begin{array}{l}\text { Detect ctDNA and confirm the presence } \\
\text { melanoma skin cancer }\end{array}$ & & {$[48]$} \\
\hline & $\begin{array}{l}\text { Detect ctDNA and confirm the presence } \\
\text { of colorectal cancer }\end{array}$ & & {$[49]$} \\
\hline & $\begin{array}{l}\text { Detect ctDNA and confirm the presence } \\
\text { of breast cancer }\end{array}$ & & {$[50]$} \\
\hline & Detect rare mutation marker & & {$[52]$} \\
\hline PCR & $\begin{array}{l}\text { Amplifying rare mutant DNA molecules } \\
\text { and detecting allele-specific mutation }\end{array}$ & $\begin{array}{l}\text { Lower sensitivity, detection } \\
\text { limit to a small number of } \\
\text { genomic positions in the sample }\end{array}$ & {$[51]$} \\
\hline $\begin{array}{l}\text { Modified next-generation } \\
\text { sequencing }\end{array}$ & $\begin{array}{c}\text { Digital error suppression-enhanced deep } \\
\text { sequencing for } 90 \% \text { of sensitivity, } 96 \% \\
\text { specificity in mutation EGFR kinase } \\
\text { domain detection }\end{array}$ & $\begin{array}{l}\text { Less comprehensive than whole } \\
\text { genome/exome sequencing }\end{array}$ & {$[55]$} \\
\hline BEAMing & Evaluate specifically RAS mutation in the blood & Evaluate only known cell mutations & {$[42]$} \\
\hline
\end{tabular}

Note: SCNA: somatic copy number alteration; NSCLC: non-small-cell lung cancer; EGRF: epidermal growth factor receptor; PCR: polymerase chain reaction; BEAMing: beads, emulsions, amplification, and magnetics.

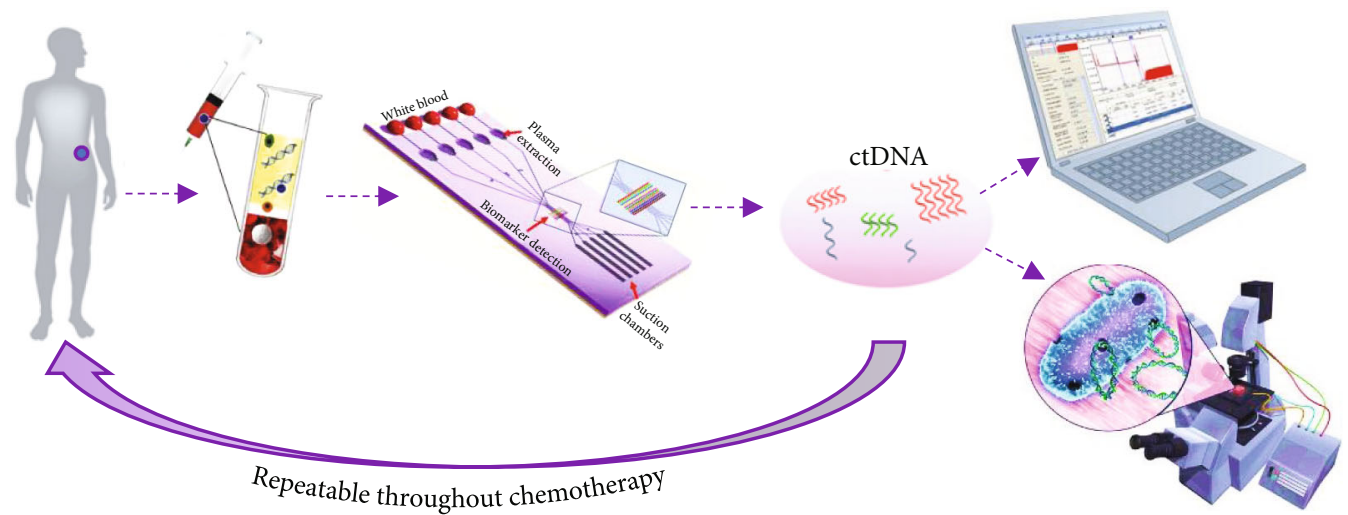

FIGURE 5: Microfluidic point of view for noninvasive liquid biopsy of ctDNA detection and analysis.

and purification. These methods have potential to overcome most of the microfluidic challenges such as improves heat dissipation, reduces cfDNA separation duration by application of large electric field, and reduces the amount of required blood sample from patient and related buffer solution for separation. One of the major limitations encountered by these methods is the separated cfDNA that could not further be purified for specific ctDNA detection. Followed by this method, concentrated ctDNA-based microfluidic particularly a narrow microchannel [60] and nanopore microchannel [61] were modified for the enrichment of ctDNA separation. Besides, another earlier development of microfluidic studies for cfDNA or ctDNA detection was based on conventional qPCR technique applied in microscale platform. Thermal amplification of a single DNA copy and real-time fluorescence was used to monitor DNA concentration with the help of intercalating dyes, e.g., SYBR Green, or DNA fluorescent probes, e.g., TaqMan. Microfluidic system with thermal amplification as qPCR and droplet-based reaction chamber which represents ddPCR were reported for cfDNA detection without sensitivity specification of the fabricated devices $[62,63]$. Chaudhuri et al. reported seven droplet-based digital PCR microfluidic system to identify somatic mutations by detection of ctDNA [64]. The droplet-based microfluidic with TaqMan ${ }^{\circledR}$ probes 


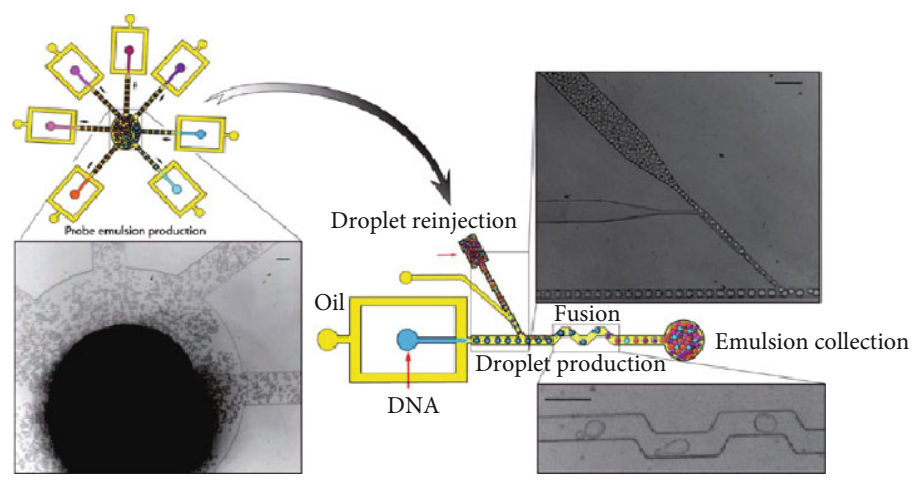

(a)
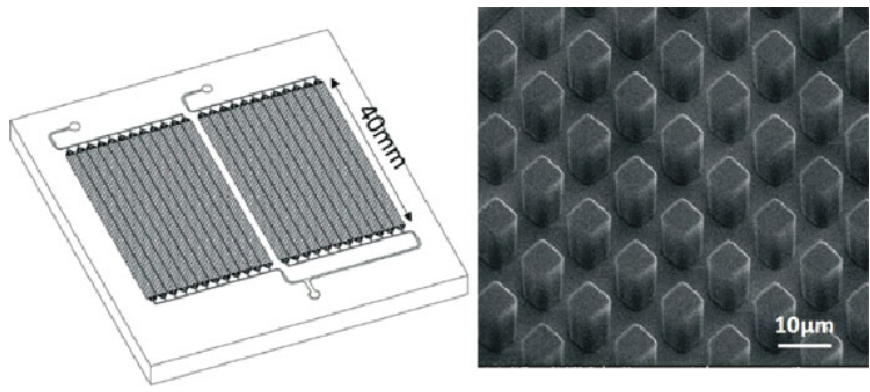

(b)

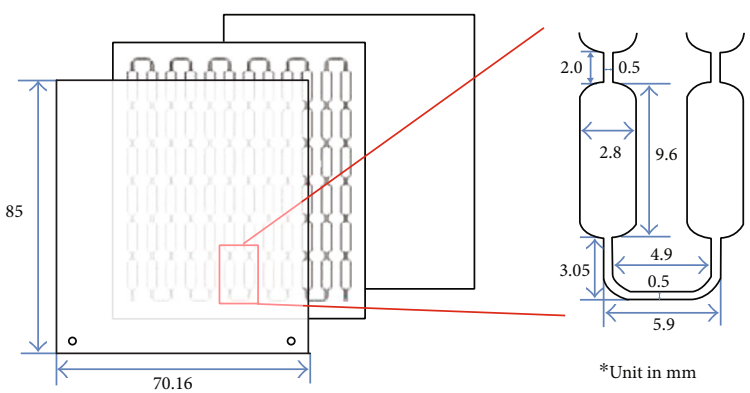

(c)

Figure 6: (a) Digital PCR-based microfluidic device with seven droplets to detect wild-type mutation of the KRAS oncogene [64]. (b) Micropillar-based plastic microfluidic surface to detect cfDNA; more than 90\% of purities of cfDNA was extracted [69]. (c) Microcolumnand microwell-based microfluidic for cfDNA isolation [71].

was used to isolate mutant DNA from wild-type DNA with fluorescent signals. Accurate and sensitive quantification of mutated KRAS oncogene was detected, although this platform is limited by the number of droplets for analysis. Bahga et al. [65] developed electrodes with dielectrophoretic capture trapping on microfluidic to separate ctDNA, and high-yields results were reported without particular details of sensitivity level of the device [66]. Microfluidic fluorescence in situ hybridization (FISH) assay was fabricated based on conventional FISH technology to screen hematopoietic malignancies. The device detected minimal residual disease can analyze 10 samples in submicroliter volume at a time [67].

To the extent of our knowledge, previous research findings on ctDNA detection by microfluidic have been limited, inconsistent, and contradictory from the past decade up to 2017. As a breakthrough, in 2017, Koboldt et al. proposed microfluidic multiplex PCR sequencing technology for high-throughput and sensitive quantitation of ctDNA
[68]. A multiplex PCR preamplification integrated with sequencing method for quantifying low concentrated ctDNA and continued by off-chip empirical Bayesian model to study error specific to characterize ctDNA; thus, precision approach can be taken to revise their work. They reported ctDNA mutation detection and disease monitoring using plasma of ovarian and pancreatic cancer patients (clinical trial samples) with sensitivity of $92 \%$ and specificity of $100 \%$ which matched with tumor tissue. A novel lowcost plastic microfluidic surface based on solid phase microextraction $(\mathrm{SP} \mu \mathrm{E})$ for cfDNA extraction was demonstrated by Campos et al. [69]. Typically, clinical analytes of interest can be extracted by solid phase extraction in microfluidic by several methods using micropillar structure, porous solid phase, or immobilized magnetic beads [70]. Campos et al. (2018) used array of micropillars to increase extraction bed load (scalable to loads $>700 \mathrm{ng}$ of cfDNA) and immobilization buffer (IB) consisting of polyethylene glycol and salts that induce cfDNA condensation onto the 
TABLE 2: Microfluidic methods for ctDNA detection to identify rare mutational marker and presence tumor.

\begin{tabular}{|c|c|c|c|}
\hline Method & Description & Limitations/achievements & References \\
\hline Electrokinetic trapping & $\begin{array}{c}\text { Separation based on properties } \\
\text { of cfDNA, depletion of cfDNA } \\
\text { based on charged ions }\end{array}$ & $\begin{array}{l}\text { cfDNA could not further purified } \\
\text { for specific ctDNA detection. }\end{array}$ & {$[57]$} \\
\hline $\begin{array}{l}\text { Micropillar, microcolumn } \\
\text { packed, electrophoretic system } \\
\text { on microchannel }\end{array}$ & Separation based on size of cfDNA & $\begin{array}{l}\text { Insensitivity of the microdevice; } \\
\text { cfDNA could not further be purified } \\
\text { for specific ctDNA detection. }\end{array}$ & {$[58,59]$} \\
\hline $\begin{array}{l}\text { ctDNA concentration-based } \\
\text { microfluidic channel }\end{array}$ & $\begin{array}{l}\text { Narrow microchannel and nanopore } \\
\text { microchannel for ctDNA separation }\end{array}$ & No sensitivity specification & {$[60,61]$} \\
\hline $\begin{array}{l}\mathrm{qPCR} \text { and droplet-based } \\
\text { reaction chamber }\end{array}$ & $\begin{array}{l}\text { Thermal amplification which } \\
\text { represents ddPCR was reported } \\
\text { for cfDNA }\end{array}$ & No sensitivity specification & {$[62,63]$} \\
\hline $\begin{array}{l}\text { Seven droplet-based digital } \\
\text { PCR microfluidic system }\end{array}$ & $\begin{array}{l}\text { Identify somatic mutations by } \\
\text { detection of ctDNA. Sensitive } \\
\text { quantification of mutated } \\
\text { KRAS oncogene }\end{array}$ & $\begin{array}{l}\text { Limited by the number of } \\
\text { droplets for analysis }\end{array}$ & {$[64]$} \\
\hline Dielectrophoretic capture & $\begin{array}{l}\text { Electrodes used for trapping on } \\
\text { microfluidic to separate ctDNA }\end{array}$ & No sensitivity specification & {$[65]$} \\
\hline Microfluidic multiplex PCR & $\begin{array}{l}\text { Integrated with sequencing technology } \\
\text { for ctDNA mutation detection and } \\
\text { disease monitoring using plasma of } \\
\text { ovarian and pancreatic cancer patients }\end{array}$ & $\begin{array}{l}\text { Sensitivity of } 92 \% \text { and } \\
\text { specificity of } 100 \%\end{array}$ & {$[68]$} \\
\hline Plastic microfluidic surface & $\begin{array}{l}\text { Micropillars and immobilization } \\
\text { buffer (IB) used for cfDNA isolation } \\
\text { from samples of colorectal and } \\
\text { non-small-cell lung cancer patients } \\
\text { to detect KRAS mutation gene }\end{array}$ & $90 \%$ of purities in cfDNA & {$[69]$} \\
\hline $\begin{array}{l}\text { Microfluidic platform } \\
\text { integrated with Sanger } \\
\text { sequencing method }\end{array}$ & $\begin{array}{c}\text { Dimethyl dithiobispropionimidate } \\
\text { (DTBP) binds to the amine group } \\
\text { of ctDNA and sodium bicarbonate } \\
\text { was used as an elution buffer } \\
\text { to isolate ctDNA. }\end{array}$ & $\begin{array}{l}\text { Identified } 71.4 \% \text { of mutation } \\
\text { profile of } K R A S \text { and } B R A F \text { from } \\
\text { colorectal cancer of patients } \\
\text { stages I to IV within } 15 \text { minutes }\end{array}$ & {$[71]$} \\
\hline
\end{tabular}

activated plastic microfluidic surface. More than $90 \%$ of purities in cfDNA extraction was noticed and the use of IB reduced the interference of coextracted genomic DNA in the final output. The chip also proved can be utilized for clinical disease detection by extracted cfDNA from plasma samples of colorectal and non-small-cell lung cancer patients to detect KRAS mutation gene. Another new method was introduced by Aravanis et al. by sampling cfDNA from blood plasma of colorectal cancer patients for ctDNA detection [71]. The microwells and microcolumn-based microfluidic platform used dimethyl dithiobispropionimidate (DTBP) for ctDNA isolation and integrated with Sanger sequencing method for ctDNA validation. The outcome from this research showed that DTBP tremendously reduces detection of cellular background particularly DNA that might be released from noncancerous cell during chemical mixing or elution process. DTBP with amine-reactive homobifunctional imidoester (HI) binds to the amine group of ctDNA and sodium bicarbonate was used as an elution buffer to isolate ctDNA. This microfluidic platform identified $71.4 \%$ of mutation profile of KRAS and $B R A F$ from colorectal cancer of patient stages I to IV within 15 minutes. Few examples of microfluidic system for isolation and detection ctDNA are shown in Figure 6.
The summary of microfluidic method for ctDNA detection is shown in Table 2.

\section{Conclusions}

The identification of suitable cancer biomarker hinges the pathway towards tangible personalized medicine. Analysis of ctDNA as cancer biomarker is amenable for noninvasive liquid biopsy which gives quick access to tumor sample rather than conventional tissue biopsy which needed surgical examination of tumor tissue. In spite of the fact that ctDNA highlighted many flourishing possibilities as well-validated prognostic and diagnostic biomarker in cancer research, yet the emergence of high sensitive and selective microfluidic device in healthcare to leverage the potential of ctDNA is still needed. As there is very limited and inconsistent research on ctDNA detection via microfluidic, a more cost-effective and time-saving microfluidic platform is necessary to cope with this era of precision medicine. In a nutshell, microfluidic analysis of ctDNA would give quick access to tumor sample rather than conventional tissue biopsy helpful in clinical setting for rapid and early ctDNA detection and appropriate treatment can be prescribed. 


\section{Future Perspectives}

Looking forward, the current challenges of microfluidic platform for ctDNA analysis are quite thrilling. Microfluidic platform for multiplex identification of biomarker has the forthcoming in quick disease detection and diagnostic. Simultaneous detection and analysis of multibiomarker would be an eye-catching approach in microfluidic research as they contain the fingerprint information of tumor or any other related disease and this feasibly improves the diagnosis accuracy. Besides, terahertz $(\mathrm{THz})$ metamaterials have unique characteristics for biosensing application which are engineered to have properties that are impossible to obtain in natural materials. However, the structures of metamaterials are enormously complicated to fabricate. $\mathrm{THz}$ metamaterial biosensor works based on tuneable resonance vibrational frequency of biomarkers and electromagnetic (EM) wave. The metamaterial is structured with optimized permittivity $\varepsilon$, conductivity $\sigma$, and dielectric material property. One of the main abilities of this biosensor is in the identification of targeted biomarker which caused hypersensitivity reaction due to the consumption of drug and antibiotic. The fluctuations in water content of biological cells and tissues display changes in the permittivity $\varepsilon$ and conductivity $\sigma$ values of cells and tissues. The variation of permittivity $\varepsilon$ and conductivity $\sigma$ values of cells in the metamaterial leads to a high-sensitive resonant frequency which can be used to detect biomarkers. Integrating $\mathrm{THz}$ metamaterial into bioanalytical device could improve the detection sensitivity and limits to single molecules.

\section{Abbreviations}

$\begin{array}{ll}\text { DNA: } & \text { Deoxyribonucleic acid } \\ \text { CTC: } & \text { Circulating tumor cell } \\ \text { ctDNA: } & \text { Circulating tumor DNA } \\ \text { RNA: } & \text { Ribonucleic acid } \\ \text { cfDNA: } & \text { Circulating cell-free DNA } \\ \text { NSCLC: } & \text { Non-small-cell lung cancer } \\ \text { PCR: } & \text { Polymerase chain reaction } \\ \text { ARMS: } & \text { Scorpion amplification refractory mutation } \\ & \text { system } \\ \text { qPCR: } & \text { Quantitative polymerase chain reaction } \\ \text { NGS: } & \text { Next-generation sequencing } \\ \text { BEAMing: } & \text { Beads, emulsions, amplification, and magnetic } \\ \text { DPCR: } & \text { Digital polymerase chain reaction } \\ \text { ddPCR: } & \text { Droplet digital polymerase chain reaction } \\ \text { EGFR: } & \text { Epidermal growth factor receptor } \\ \text { TKI: } & \text { Tyrosine kinase inhibitors } \\ \text { WGS: } & \text { Whole genome sequencing } \\ \text { WES: } & \text { Whole exome sequencing } \\ \text { TAm-Seq: } & \text { Tagged-amplicon deep sequencing } \\ \text { Safe-Seq: } & \text { Safe-sequencing system } \\ \text { FISH: } & \text { Fluorescence in situ hybridization } \\ \text { SP } \mu \text { E: } & \text { Solid phase microextraction } \\ \text { IB: } & \text { Immobilization buffer } \\ \text { DTBP: } & \text { Dimethyl dithiobispropionimidate } \\ \text { HI: } & \text { Homobifunctional imidoester } \\ \text { THz: } & \text { Terahertz. } \\ & \end{array}$

\section{Conflicts of Interest}

Authors declare no conflict of interest.

\section{Acknowledgments}

The research was supported by the Ministry of Higher Education of Malaysia and Universiti Teknologi Malaysia (grant nos. Q.J130000.21A2.04E82 and Q.J130000.2523.16H38). We thank them for funding this project and for their endless support.

\section{References}

[1] World Health Organization \& International Programme on Chemical Safety, "Biomarkers in risk assessment : validity and validation," in Relation Environmental Health Criteria, vol. 222, p. 238, World Health Organization, 2001, https:// apps.who.int/iris/handle/10665/42363.

[2] N. Goossens, S. Nakagawa, X. Sun, and Y. Hoshida, "Cancer biomarker discovery and validation," Translational Cancer Research, vol. 4, no. 3, pp. 256-269, 2015.

[3] N. L. Henry and D. F. Hayes, "Cancer biomarkers," Molecular Oncology, vol. 6, no. 2, pp. 140-146, 2012.

[4] F. S. Collins and H. Varmus, "A new initiative on precision medicine," The New England Journal of Medicine, vol. 372, no. 9, pp. 793-795, 2015.

[5] M. T. Gabriel, L. R. Calleja, A. Chalopin, B. Ory, and D. Heymann, "Circulating tumor cells: a review of nonEpCAM-based approaches for cell enrichment and isolation," Clinical Chemistry, vol. 62, no. 4, pp. 571-581, 2016.

[6] P. Potdar and N. Lotey, "Role of circulating tumor cells in future diagnosis and therapy of cancer," Journal of Cancer Metastasis and Treatment, vol. 1, no. 2, p. 44, 2015.

[7] T. A. Yap, D. Lorente, A. Omlin, D. Olmos, and J. S. De Bono, "Circulating tumor cells: a multifunctional biomarker," Clinical Cancer Research, vol. 20, no. 10, pp. 2553-2568, 2014.

[8] K. L. Georgiadis, K. Simpson, M. Ayub et al., "Circulating tumor cells," in Pancreatic Cancer, pp. 1325-1360, Springer, New York, 2018.

[9] C. Alix-Panabières and J.-Y. Pierga, "Circulating Tumor Cells : Liquid Biopsy,” Bulletin du Cancer, vol. 101, no. 1, pp. 17-23, 2014.

[10] X. Zhe, M. L. Cher, and R. D. Bonfil, "Circulating tumor cells: finding the needle in the haystack," American Journal of Cancer Research, vol. 1, no. 6, pp. 740-751, 2011.

[11] J. D. Merker, G. R. Oxnard, C. Compton et al., "Circulating tumor DNA analysis in patients with cancer: American society of clinical oncology and college of American pathologists joint review," Archives of Pathology \& Laboratory Medicine, vol. 142, no. 10, pp. 1242-1253, 2018.

[12] C. M. Stewart, P. D. Kothari, F. Mouliere et al., "The value of cell-free DNA for molecular pathology," The Journal of Pathology, vol. 244, no. 5, pp. 616-627, 2018.

[13] S. Volik, M. Alcaide, R. D. Morin, and C. Collins, "Cell-free DNA (cfDNA): clinical significance and utility in cancer shaped by emerging technologies," Molecular Cancer Research, vol. 14, no. 10, pp. 898-908, 2016.

[14] J. Liu, X. Chen, J. Wang et al., "Biological background of the genomic variations of cf-DNA in healthy individuals," Annals of Oncology, vol. 30, no. 3, pp. 464-470, 2019. 
[15] M. Kleppe and R. L. Levine, “Tumor heterogeneity confounds and illuminates: assessing the implications," Nature Medicine, vol. 20, no. 4, pp. 342-344, 2014.

[16] L. De Mattos-Arruda, B. Weigelt, J. Cortes et al., "Capturing intra-tumor genetic heterogeneity by de novo mutation profiling of circulating cell-free tumor DNA: a proof-of-principle," Annals of Oncology, vol. 25, no. 9, pp. 1729-1735, 2014.

[17] M. Murtaza, S. J. Dawson, K. Pogrebniak et al., "Multifocal clonal evolution characterized using circulating tumour DNA in a case of metastatic breast cancer," Nature Communications, vol. 6, no. 1, 2015.

[18] M. Yang, M. E. Forbes, R. L. Bitting et al., "Incorporating blood-based liquid biopsy information into cancer staging: time for a TNMB system?," Annals of Oncology, vol. 29, no. 2, pp. 311-323, 2018.

[19] E. I. Deryugina and W. B. Kiosses, "Intratumoral cancer cell intravasation can occur independent of invasion into the adjacent stroma," Cell Reports, vol. 19, no. 3, pp. 601-616, 2017.

[20] E. Yong, "Cancer biomarkers: written in blood," Nature, vol. 511, no. 7511, pp. 524-526, 2014.

[21] C. Bettegowda, M. Sausen, R. J. Leary et al., "Detection of circulating tumor DNA in early- and late-stage human malignancies," Science Translational Medicine, vol. 6, no. 224, p. 224ra24, 2014.

[22] E. Crowley, F. Di Nicolantonio, F. Loupakis, and A. Bardelli, "Liquid biopsy: monitoring cancer-genetics in the blood," Nature Reviews Clinical Oncology, vol. 10, no. 8, pp. 472-484, 2013.

[23] B. S. Sorensen, L. Wu, W. Wei et al., "Monitoring of epidermal growth factor receptor tyrosine kinase inhibitor-sensitizing and resistance mutations in the plasma DNA of patients with advanced non-small cell lung cancer during treatment with erlotinib," Cancer, vol. 120, no. 24, pp. 3896-3901, 2014.

[24] E. S. Gray, H. Rizos, A. L. Reid et al., "Circulating tumor DNA to monitor treatment response and detect acquired resistance in patients with metastatic melanoma," Oncotarget, vol. 6, no. 39, pp. 42008-42018, 2015.

[25] T. Reinert, L. V. Schøler, R. Thomsen et al., "Analysis of circulating tumour DNA to monitor disease burden following colorectal cancer surgery," Gut, vol. 65, no. 4, pp. 625634, 2016.

[26] S. V. Bratman, A. M. Newman, A. A. Alizadeh, and M. Diehn, "Potential clinical utility of ultrasensitive circulating tumor DNA detection with CAPP-Seq," Expert Review of Molecular Diagnostics, vol. 15, no. 6, pp. 715-719, 2015.

[27] C. K. Y. Ng, G. G. Di Costanzo, N. Tosti et al., "Genetic profiling using plasma-derived cell-free DNA in therapy-naïve hepatocellular carcinoma patients: a pilot study," Annals of Oncology, vol. 29, no. 5, pp. 1286-1291, 2018.

[28] K. Sun, P. Jiang, K. C. A. Chan et al., "Plasma DNA tissue mapping by genome-wide methylation sequencing for noninvasive prenatal, cancer, and transplantation assessments," Proceedings of the National Academy of Sciences, vol. 112, no. 40, pp. E5503-E5512, 2015.

[29] L. D. Moore, T. Le, and G. Fan, "DNA methylation and its basic function," Neuropsychopharmacology, vol. 38, no. 1, pp. 23-38, 2013.

[30] C. K. Y. Ng, G. G. Di Costanzo, L. M. Terracciano, and S. Piscuoglio, "Circulating cell-free DNA in hepatocellular carcinoma: current insights and outlook," Frontiers in Medicine, vol. 5, 2018.
[31] K. Kichko, P. Marschall, and S. Flessa, "Personalized medicine in the U.S. and Germany: awareness, acceptance, use and preconditions for the wide implementation into the medical standard," Journal of Personalized Medicine, vol. 6, no. 2, p. 15, 2016.

[32] S. Abou Daya and R. Mahfouz, "Circulating tumor DNA, liquid biopsy, and next generation sequencing: a comprehensive technical and clinical applications review," Meta Gene, vol. 17, pp. 192-201, 2018.

[33] M. H. D. Neumann, S. Bender, T. Krahn, and T. Schlange, "ctDNA and CTCs in Liquid Biopsy - Current Status and Where We Need to Progress," Computational and Structural Biotechnology Journal, vol. 16, pp. 190-195, 2018.

[34] L. Sorber, K. Zwaenepoel, V. Deschoolmeester et al., "Circulating cell-free nucleic acids and platelets as a liquid biopsy in the provision of personalized therapy for lung cancer patients," Lung Cancer, vol. 107, pp. 100-107, 2017.

[35] H. Li, C. Jing, J. Wu et al., "Circulating tumor DNA detection: a potential tool for colorectal cancer management (Review)," Oncology Letters, vol. 17, pp. 1409-1416, 2018.

[36] B. Zhang, C.-W. Xu, Y. Shao et al., "Comparison of droplet digital PCR and conventional quantitative PCR for measuring EGFR gene mutation," Experimental and Therapeutic Medicine, vol. 9, no. 4, pp. 1383-1388, 2015.

[37] Z. Shaozhang, Z. Ming, P. Haiyan, Z. Aiping, Y. Qitao, and S. Xiangqun, "Comparison of ARMS and direct sequencing for detection of EGFR mutation and prediction of EGFR-TKI efficacy between surgery and biopsy tumor tissues in NSCLC patients," Medical Oncology, vol. 31, no. 5, pp. 1-9, 2014.

[38] J. Liu, R. Zhao, J. Zhang, and J. Zhang, "ARMS for EGFR mutation analysis of cytologic and corresponding lung adenocarcinoma histologic specimens," Journal of Cancer Research and Clinical Oncology, vol. 141, no. 2, pp. 221227, 2015.

[39] A. A. Chaudhuri, M. S. Binkley, E. C. Osmundson, A. A. Alizadeh, and M. Diehn, "Predicting radiotherapy responses and treatment outcomes through analysis of circulating tumor DNA," Seminars in Radiation Oncology, vol. 25, no. 4, pp. 305-312, 2015.

[40] F. Cheng, L. Su, and C. Qian, "Circulating tumor DNA: a promising biomarker in the liquid biopsy of cancer," Oncotarget, vol. 7, no. 30, pp. 48832-48841, 2016.

[41] J. C. M. Wan, C. Massie, J. Garcia-Corbacho et al., "Liquid biopsies come of age: towards implementation of circulating tumour DNA," Nature Reviews. Cancer, vol. 17, no. 4, pp. 223-238, 2017.

[42] J. García-Foncillas, E. Alba, E. Aranda et al., "Incorporating BEAMing technology as a liquid biopsy into clinical practice for the management of colorectal cancer patients: an expert taskforce review," Annals of Oncology, vol. 28, no. 12, pp. 2943-2949, 2017.

[43] D. C. Koboldt, K. Chen, T. Wylie et al., "VarScan: variant detection in massively parallel sequencing of individual and pooled samples," Bioinformatics, vol. 25, no. 17, pp. 22832285, 2009.

[44] J. A. Reuter, D. V. Spacek, and M. P. Snyder, "High-throughput sequencing technologies," Molecular Cell, vol. 58, no. 4, pp. 586-597, 2015.

[45] J. C. Thompson, S. S. Yee, A. B. Troxel et al., "Detection of therapeutically targetable driver and resistance mutations in lung cancer patients by next-generation sequencing of cell- 
free circulating tumor DNA," Clinical Cancer Research, vol. 22, no. 23 , pp. 5772-5782, 2016.

[46] A. M. Aravanis, M. Lee, and R. D. Klausner, "Next-generation sequencing of circulating tumor DNA for early cancer detection,” Cell, vol. 168, no. 4, pp. 571-574, 2017.

[47] X. Han, J. Wang, and Y. Sun, "Circulating Tumor DNA as Biomarkers for Cancer Detection," Genomics, Proteomics \& Bioinformatics, vol. 15, no. 2, pp. 59-72, 2017.

[48] H. Ishii, K. Azuma, K. Sakai et al., "Digital PCR analysis of plasma cell-free DNA for non-invasive detection of drug resistance mechanisms in EGFR mutant NSCLC: correlation with paired tumor samples," Oncotarget, vol. 6, no. 31, pp. 3085030858, 2015.

[49] J. A. Denis, A. Patroni, E. Guillerm et al., "Droplet digital PCR of circulating tumor cells from colorectal cancer patients can predict KRAS mutations before surgery," Molecular Oncology, vol. 10, no. 8, pp. 1221-1231, 2016.

[50] D. Chu, C. Paoletti, C. Gersch et al., "ESR1 mutations in circulating plasma tumor DNA from metastatic breast cancer patients," Clinical Cancer Research, vol. 22, no. 4, pp. 993999, 2016.

[51] A. Ashida, K. Sakaizawa, A. Mikoshiba, H. Uhara, and R. Okuyama, "Quantitative analysis of the BRAFV600E mutation in circulating tumor-derived DNA in melanoma patients using competitive allele-specific TaqMan PCR," International Journal of Clinical Oncology, vol. 21, no. 5, pp. 981-988, 2016.

[52] J. H. van Ginkel, D. A. van den Broek, J. van Kuik et al., "Preanalytical blood sample workup for cell-free DNA analysis using Droplet Digital PCR for future molecular cancer diagnostics," Cancer Medicine, vol. 6, no. 10, pp. 2297-2307, 2017.

[53] E. Heitzer, P. Ulz, J. Belic et al., "Tumor-associated copy number changes in the circulation of patients with prostate cancer identified through whole-genome sequencing," Genome Medicine, vol. 5, no. 4, p. 30, 2013.

[54] A. M. Newman, S. V. Bratman, Jacqueline To et al., "An ultrasensitive method for quantitating circulating tumor DNA with broad patient coverage," Nature Medicine, vol. 20, no. 5, pp. 548-554, 2014.

[55] A. M. Newman, A. F. Lovejoy, D. M. Klass et al., "Integrated digital error suppression for improved detection of circulating tumor DNA," Nature Biotechnology, vol. 34, no. 5, pp. 547$555,2016$.

[56] E. Bohers, P. J. Viailly, S. Dubois et al., "Somatic mutations of cell-free circulating DNA detected by next-generation sequencing reflect the genetic changes in both germinal center B-cell-like and activated B-cell-like diffuse large B-cell lymphomas at the time of diagnosis," Haematologica, vol. 100, no. 7, pp. e280-e284, 2015.

[57] T. Hahn, C. K. O’Sullivan, and K. S. Drese, "Microsystem for field-amplified electrokinetic trapping preconcentration of DNA at poly(ethylene terephthalate) membranes," Analytical Chemistry, vol. 81, no. 8, pp. 2904-2911, 2009.

[58] M. Sun, J. S. Lin, and A. E. Barron, "Ultrafast, efficient separations of large-sized dsDNA in a blended polymer matrix by microfluidic chip electrophoresis: a design of experiments approach," Electrophoresis, vol. 32, no. 22, pp. 3233-3240, 2011.

[59] A. Barry and S. Cohen, "Quality assessment of genomic DNA degradation," Genetic Engineering \& Biotechnology News, vol. 33 , no. 16, p. $28,2013$.
[60] C. H. Kuo, J. H. Wang, and G. B. Lee, “A microfabricated CE chip for DNA pre-concentration and separation utilizing a normally closed valve," Electrophoresis, vol. 30, no. 18, pp. 3228-3235, 2009.

[61] K. H. Paik, Y. Liu, V. Tabard-Cossa et al., "Control of DNA capture by nanofluidic transistors," ACS Nano, vol. 6, no. 8, pp. 6767-6775, 2012.

[62] B. Vogelstein and K. W. Kinzler, "Digital PCR," Proceedings of the National Academy of Sciences, vol. 96, no. 16, pp. 92369241, 1999.

[63] M. Baker, "Digital PCR hits its stride," Nature Methods, vol. 9, no. 6, pp. 541-544, 2012.

[64] D. Pekin, Y. Skhiri, J.-C. Baret et al., "Quantitative and sensitive detection of rare mutations using droplet-based microfluidics," Lab on a Chip, vol. 11, no. 13, pp. 2156-2166, 2011.

[65] S. S. Bahga, C. M. Han, and J. G. Santiago, "Integration of rapid DNA hybridization and capillary zone electrophoresis using bidirectional isotachophoresis," Analyst, vol. 138, no. 1, pp. 87-90, 2013.

[66] R. Malbec, J. Cacheux, P. Cordelier, T. Leichlé, P. Joseph, and A. Bancaud, "Microfluidics for minute DNA sample analysis: open challenges for genetic testing of cell-free circulating DNA in blood plasma," Micro and Nano Engineering, vol. 1, pp. 25-32, 2018.

[67] F. Mughal, S. J. Baldock, E. G. Karimiani, N. Telford, N. J. Goddard, and P. J. R. Day, "Microfluidic channel-assisted screening of hematopoietic malignancies," Genes, Chromosomes and Cancer, vol. 53, no. 3, pp. 255-263, 2014.

[68] Y. Guan, O. Mayba, T. Sandmann et al., "High-throughput and sensitive quantification of circulating tumor DNA by microfluidic-based multiplex PCR and next-generation sequencing," The Journal of Molecular Diagnostics, vol. 19, no. 6, pp. 921-932, 2017.

[69] C. D. M. Campos, S. S. T. Gamage, J. M. Jackson et al., "Microfluidic-based solid phase extraction of cell free DNA," Lab on a Chip, vol. 18, no. 22, pp. 3459-3470, 2018.

[70] G. Samla, K. B. Gan, and S. M. Then, "Solid phase microextraction based micro-device for extraction of PCR amplifiable DNA," International Journal of Nanoelectronics and Materials, vol. 10, pp. 63-80, 2017.

[71] C. E. Jin, B. Koo, T. Y. Lee et al., "Simple and low-cost sampling of cell-free nucleic acids from blood plasma for rapid and sensitive detection of circulating tumor DNA," Advancement of Science, vol. 5, no. 10, 2018. 